\title{
ASSOCIAÇÕES ENTRE AUTO-CONCEITO FÍSICO E MOTIVAÇÃO PARA O EXERCÍCIO EM ADOLESCENTES: INTERACÇÕES COM O NÍVEL DE PRÁTICA E O GÉNERO
}

\author{
Associations between physical-self and exercise motivation in \\ adolescents: interactions with physical activity level and gender
}

\author{
Mónica Silva ${ }^{1}$; António Labisa Palmeira1 \\ ${ }^{1}$ Centro de Estudos e Intervenção em Educação e Formação \\ Faculdade de Educação Física e Desporto \\ Universidade Lusófona de Humanidades e Tecnologias
}

\begin{abstract}
Resumo: Este estudo analisou o auto-conceito físico e a importância atribuída ao auto-conceito em adolescentes, estudando a sua associação com a motivação para a prática de actividade física e verificando se varia consoante o nível de actividade física e género. Método: Participaram neste estudo 460 estudantes (223 do sexo feminino e 237 do sexo masculino, M=16.60 e DP=1.18 anos). Utilizaram-se os instrumentos Behavioral Regulation in Exercise Questionnaire - 2 (BREQ-2, Palmeira et al, 2007, .58<alfa<.83), para avaliação motivação para o exercício e o Physical SelfPerception Profile R (PSPP-R, Palmeira et al, 2009, .75<alfa<.89), para avaliação do auto-conceito físico. O estudo comparativo foi realizado através da MANOVA, enquanto que o estudo correlacional foi efectuado através da correlação de Pearson. Resultados: Os adolescentes que praticam desporto federado tem valores significativamente superiores aos restantes grupos (desporto não federado e só Educação Física) na maioria das dimensões do auto-conceito físico, importância atribuída às dimensões de auto-conceito e motivação. Os moços registaram valores mais elevados no auto-conceito físico (com excepção do corpo atractivo onde não se registaram diferenças) e motivação quando comparados com as moças ( $p<.05$ ). No entanto, as moças e moços do grupo que pratica desporto federado apresentam valores muito próximos de auto-conceito físico e motivação. As regulações motivacionais menos auto-determinadas apresentaram associações negativas com o auto-conceito físico, enquanto que as mais autodeterminadas revelaram associações positivas $(p<.05)$. Conclusões: Este estudo sugere que quanto maior o nível de prática, mais elevados são os valores de auto-conceito físico, importância atribuída às dimensões do auto-conceito e motivação, o que deve promover a manutenção da prática de actividade física no longo-prazo.
\end{abstract}

Palavras Chave: auto-conceito físico; auto-estima; motivação para o exercício; actividade física; adolescentes.

Abstract: Purpose: This study focused on adolescent's physical-self, analyzing its association with the motivation for physical activity while verifying if it changes in function of gender and level of activity. Method: Participants were 460 adolescents ( 223 girls and 237 boys, $M=16.60, S D=1.18$ years). Exercise motivation was measured with the Behavioral Regulation in Exercise Questionnaire - 2 (BREQ-2, Palmeira et al, 2007, .58<alpha<.83) and the physical-self was assessed with the Physical Self-Perception Profile - R (PSPP-R, Palmeira et al, 2009, .75<alpha<.89). The comparisons were evaluated through a MANOVA, whilst Pearson correlations were used for the correlation analysis. Results: The adolescents that compete at the federation level had higher scores on most dimensions of the physical-self, perception of importance for the physical-self and exercise motivation, when compared with the adolescents that did not compete or only did the physical education. Boys had higher values of the physical-self (except body attractiveness) and motivation when compared to girls $(p<.05)$. Notwithstanding, boys and girls' values of physical-self and motivation were similar on the group that played competitive sport. The less self-determined behavioral regulations where negatively associated with the physicalself. Conversely, the most self-determined had positive association $(p<.05)$. Conclusions: This study suggests that the higher levels of engagement with physical activity (e.g., competitive sport) are associated with self-determined motivations and physical-self, which should benefit the long-term maintenance of the physical activity engagement.

Key Words: physical self; self-esteem; exercise motivation; physical activity; adolescents.

Aceito em 25/05/2010 - Rev. Educ. Fís. 2010 - 27-36. Rio de Janeiro - RJ - Brasil

\section{INTRODUÇÃO}

Os benefícios da actividade física na saúde são amplamente conhecidos e representam um importante mecanismo de promoção da saúde pública, especialmente importante numa fase de desenvolvimento tão acentuada como a adolescência $^{(1)}$. Estes benefícios são evidentes em todas as dimensões da saúde, sejam elas de âmbito fisiológico, psicológico ou social, sendo estas últimas, no entanto, menos trabalhadas na literatura científica e, por isso mesmo, com menor evidência disponível acerca dos mecanismos pelos quais a actividade física regular pode promover 
este tipo de benefícios psicossociais ${ }^{(2)}$.

Pese embora este reconhecimento, os níveis de prática de actividade física regular entre os adolescentes não parece suficiente para que se possam usufruir dos seus benefícios. Recentemente, foi efectuado um levantamento dos níveis de aptidão física e de composição corporal numa amostra representativa da população adolescente portuguesa. A amostra foi constituída por 22048 crianças e adolescentes (11 373 moças e 10675 moços) entre os 10 e os 18 anos de idade. Os resultados deste estudo mostraram que $59.3 \%$ das moças estão dentro da zona saudável da aptidão cardio-respiratória. No entanto, verificouse que estes valores decresciam nos escalões etários mais elevados. Nos moços verificou-se que $63.1 \%$ são considerados aptos na aptidão cardiorespiratória, não se registando uma assimetria tão acentuada como nas moças nos diferentes escalões etários. No que respeita à composição corporal, verificou-se que $22.4 \%$ apresentam excesso de peso ou obesidade $(17,2 \%$ de excesso de peso e $5,2 \%$ obesidade), sendo que $21.1 \%$ das moças e $23.3 \%$ dos moços têm excesso de peso ou obesidade ${ }^{(3)}$.

Estes resultados colocam a população adolescente portuguesa entre as que apresentam maiores prevalências de excesso de peso e obesidade da Europa, valores que são acompanhados pelos resultados abaixo da média na aptidão cardiorespiratória e níveis de actividade física ${ }^{(4)}$, revelando que, potencialmente, os efeitos benéficos da prática regular de actividade física não estarão a ser alcançados pela maior parte dos jovens portugueses.

Paradoxalmente, nesta fase da adolescência regista-se um desenvolvimento acelerado das estruturas biológicas do corpo humano, que são acompanhadas pela definição de diversos aspectos das características psicossociais que acompanharam o jovem para o resto da sua vida $^{(1)}$. É nesta fase que o corpo dos moços naturalmente ganha massa muscular e que adquirem conhecimentos de idealização de uma imagem corporal muscular ideal, enquanto que as raparigas ganham mais massa gorda e idealizam a imagem corporal de um corpo elegante ${ }^{(5)}$, pelo que a prática da actividade física poderia potenciar um desenvolvimento mais consistente dos nossos jovens. Adicionalmente, a sociedade actual exerce cada vez mais pressão nos indivíduos relativamente à idealização da imagem corporal $^{(6)}$, pelo que importa conhecer as associações que se estão a criar entre a actividade física e o desenvolvimento psicossocial dos jovens, num cenário onde existe uma prevalência de inactividade acentuada, contrastando com alguns jovens que, porventura, se integram em actividades desportivas com maior regularidade (por exemplo, em desporto federado ou escolar - competitivo - ou desporto de lazer, sem um quadro competitivo definido mas com uma prática regular - surf, caminhadas, natação por lazer).

$\mathrm{Na}$ revisão de literatura efectuada, duas variáveis salientaram-se como mecanismos que potencialmente irão ter um papel na procura de conhecer as associações entre a actividade física e o desenvolvimento psicossocial. Elas foram o auto-conceito corporal $^{(7)}$ e a motivação para a prática de actividade física ${ }^{(8)}$.

O auto-conceito é referenciado na literatura como um tema de interesse nas últimas décadas, sendo necessário cada vez mais tomar conhecimento da percepção que cada um tem de si próprio, especialmente pertinente na adolescência - etapa fundamental do nosso desenvolvimento ${ }^{(7,9)}$. As pesquisas no tema do auto-conceito devem-se ao desenvolvimento de instrumentos que respeitam o modelo hierárquico multidimensional do autoconceito $^{(10)}$, como o Physical Self-Perception Profile (PSPP) acompanhado pelo Perceived Importance Profile (PIP). O PIP foi desenvolvido para avaliar a importância que os indivíduos atribuem às quatro dimensões do PSPP, competência desportiva, condição física, corpo atraente e força física ${ }^{(11)}$.

Os estudos realizados com adolescentes têm revelado que os valores mais elevados de auto-conceito estão associados aos níveis mais elevados de actividade física ${ }^{(12,13)} \mathrm{e}$, em estudos experimentais, ao aumento de prática de actividade física ${ }^{(14)}$. Esta temática apresenta um interesse crescente, devido às pressões sociais a que cada vez mais os adolescentes estão sujeitos, o que pode condicionar o seu desenvolvimento harmonioso ${ }^{(6)}$.

Dada a natureza física do exercício, seria surpreendente se a percepção corporal não influenciasse os motivos de participação que os indivíduos adoptam. A literatura científica mostra associações entre a imagem corporal e os motivos 
de participação na actividade física ${ }^{(15,16)}$. Mais recentemente, Ingledew e Sullivan ${ }^{(17)}$, elaboraram um estudo com o objectivo de examinar as diferenças nos efeitos da massa corporal e imagem corporal entre idade e género e motivos para a prática de exercício, com uma amostra de 180 adolescentes de ambos os sexos, comparando dois grupos de adolescentes, mais novos ${ }^{(11-13 \text { anos })}$ e mais velhos ${ }^{(17-19 \text { anos })}$, através da análise de vários elementos: participação no exercício, motivos para o exercício, percepção corporal e forma corporal ideal, peso e estatura, chegando à confirmação de que as diferenças entre géneros são notórias durante a adolescência. Noutro trabalho, objectivou-se examinar o modelo teórico da autoestima global incorporando constructos das teorias da orientação motivacional e da auto-determinação nas aulas de educação física, com uma amostra de 634 estudantes com idades compreendidas entre os 11 e os 15 anos $^{(18)}$. Os resultados sugeriram que as orientações motivacionais influenciam a autoestima afectando a motivação conseguida pela autonomia. Este facto é consistente com a teoria que sugere que as experiências relacionadas com a motivação intrínseca são o mecanismo pelo qual a orientação motivacional global é transformada em efeitos adaptados como a auto-estima. Os resultados sugerem ainda que para os professores de educação física aumentarem a auto-estima das crianças nas suas aulas, devem proporcionar um bom clima de aula que suporte a autonomia dos alunos e orientar os alunos tanto para a tarefa como para o ego(18). Por último, Wilson e Rodgers $^{(19)}$, num estudo efectuado com o propósito de examinar as relações entre os motivos para o exercício e a auto-estima corporal, utilizando uma amostra de 114 indivíduos do sexo feminino, todas praticantes de exercício físico regular, verificaram que as mulheres que apresentaram valores mais elevados de motivação por regulação identificada e intrínseca indicaram ter percepções mais elevadas da sua auto-estima corporal.

Estes conceitos associados à motivação utilizados nos últimos trabalhos apresentados, resultam da teoria da auto-determinação ${ }^{(20)}$, que é um racional teórico usado extensamente na literatura e que a motivação intrínseca assume um papel essencial na manutenção da prática de actividade física a longo prazo. Mas poucos estudos têm sido efectuados com adolescentes, verificando-se que os principais resultados sugerem que se o exercício for realizado apenas por razões extrínsecas, sem prazer e interesse pela actividade escolhida dificilmente se a conseguirá manter com a regularidade necessária para a obtenção dos benefícios que lhe são reconhecidos ${ }^{(21,22)}$. Esta teoria prevê que a qualidade da motivação é essencial, proporcionando diferentes formas de regular o comportamento, resultando de um continuum motivacional (amotivação - extrínseca - intrínseca). Esta tendência é facilitada quando o próprio ambiente social proporciona suporte para as três necessidades psicológicas básicas, autonomia, competência e relação. Por conseguinte, a mensagem que envolve a promoção do exercício é de que providenciando esse suporte irá encorajar os indivíduos ${ }^{(20)}$.

A teoria da auto-determinação discute ainda a relação entre a auto-estima global e o comportamento motivacional e sugere que a auto-estima pode ser desenvolvida através de comportamentos regulados autonomamente e que proporcionem sentimentos de competência e de relação ${ }^{(23)}$. No entanto, a teoria não discute a relação entre a auto-estima corporal e comportamento motivacional, mas os autores Thogersen-Ntoumani e Ntoumanis ${ }^{(24)}$, acreditam que as regulações motivacionais da auto-determinação podem estar relacionadas positivamente com a auto-estima corporal, baseados nos argumentos de Fox $^{(7)}$ que indicou que a auto-determinação pode actuar como um mecanismo mediador na relação entre exercício e auto-estima corporal.

Sendo esta uma temática recorrente na literatura internacional, que não tem, segundo a nossa pesquisa, paralelo na população lusófona, considerámos interessante efectuar um estudo com adolescentes que analise o auto-conceito, a importância que estes atribuem às dimensões do auto-conceito e também a motivação para a prática de actividade física, tendo em conta o nível de prática de actividade e o género.

\section{Objectivo do Estudo}

Com este estudo pretendemos analisar o auto-conceito e a importância atribuída ao autoconceito em adolescentes, estudando a sua associação com a motivação para a prática de actividade física e verificando se varia consoante o nível de actividade física e género. 


\section{MÉTODO}

\section{Amostra}

Participaram neste estudo 460 adolescentes de ambos os sexos, 223 do sexo feminino e 237 do sexo masculino, com idades compreendidas entre os 14 e os 18 anos ( $M=16.60$ e $D P=1.18)$. Todos os indivíduos deste estudo eram estudantes do ensino secundário de uma escola na região de Lisboa (ver TABELA 1).

TABELA 1. Características demográficas da amostra do estudo.

\begin{tabular}{|c|c|c|c|c|c|c|}
\hline & \multicolumn{3}{|c|}{ Feminino } & \multicolumn{3}{|c|}{ Masculino } \\
\hline $\mathrm{N}(\%)$ & & $\begin{array}{c}237 \\
(51.5 \%)\end{array}$ & & & $\begin{array}{c}223 \\
(48.5 \%)\end{array}$ & \\
\hline \multirow[t]{2}{*}{ Idade $(M \pm D P)$} & & $16.49 \pm 1.04$ & & & $16.71 \pm 1.31$ & \\
\hline & $\overline{E F}$ & EFMNF & EFD & $\mathrm{EF}$ & EFMNF & EFD \\
\hline $\mathrm{N}(\%)$ & $\begin{array}{c}150 \\
(32.6 \%)\end{array}$ & $50(10.9 \%)$ & $37(8.0 \%)$ & 91 (19.8\%) & $59(12.8 \%)$ & $73(15.9 \%)$ \\
\hline $\begin{array}{l}\text { Idade } \\
(M \pm D P)\end{array}$ & $16.45 \pm 1.05$ & $16.58 \pm 1.03$ & $16.51 \pm 1.07$ & $16.80 \pm 1.31$ & $16.61 \pm 1.46$ & $16.68 \pm 1.15$ \\
\hline
\end{tabular}

Nota: EF - Educação Física; EFMNF - Educação Física mais Modalidade Desportiva Não Federada; EFD - Educação Física mais Desporto (ver texto para mais informações)

Os adolescentes foram divididos em grupos de prática conforme: a) realizavam só a disciplina de Educação Física na escola (obrigatória em Portugal nestes anos de ensino); b) realizavam a Educação Física mais uma modalidade desportiva não federada (enquadrámos aqui os praticantes de modalidades desportivas sem quadro competitivo, em que a prática está associada ao lazer e à promoção da saúde: surf, academias de fitness, etc); e c) realizavam a Educação Física e uma modalidade desportiva federada, com quadro competitivo (com uma regularidade de treino e exigência superior, portanto).

\section{Instrumentos}

Caracterização dos estudantes

A primeira parte do questionário foi elaborada especificamente para este estudo, com o propósito de caracterizar os estudantes com questões relacionadas com a idade, peso, altura, ano de escolaridade, género, quantas vezes tem educação física por semana, qual a duração de cada aula e qual foi a nota de educação física no período passado. No questionário entregue aos alunos que praticavam apenas educação física e/ou outra modalidade não federada questionou-se ainda: se pratica alguma actividade física regularmente; se sim, qual; quantas vezes por semana; qual a duração de cada sessão/treino e há quantos anos pratica. No questionário entregue aos alunos, que para além da educação física ainda praticavam desporto escolar ou desporto federado foram ainda efectuadas as seguintes questões: faz parte de uma equipa de desporto escolar; se sim, de que modalidade; quantas vezes por semana; qual a duração dos treinos, há quantos anos pratica; pratica algum desporto federado; se sim, qual a modalidade; qual o nível competitivo; quantas vezes por semana; qual a duração dos treinos e há quantos anos pratica.

Motivação-Behavioural Regulation in Exercise Questionnaire - 2 (BREQ-2)

Na segunda parte do questionário, foi utilizado o instrumento Behavioral Regulation in Exercise Questionnaire-2 (BREQ-2), elaborado por Markland e Tobin $^{(25)}$, traduzido para a língua portuguesa ${ }^{(26)}$ e é originalmente composto por dezanove itens relativas à motivação, com uma escala de Likert de 5 pontos, avaliando 5 dimensões: amotivação, regulação externa, regulação introjectada, regulação identificada e motivação intrínseca. Cada um dos itens foi respondido de zero a quatro, em que o zero significa "não é verdade para mim" até "muitas vezes é verdade para mim". A consistência interna das dimensões na aplicação no nosso estudo foi satisfatória (amotivação, $\alpha=0.67$; regulação externa, $\alpha=0.68$, introjectada, $\alpha=0.64$; identificada, $\alpha=0.58$; motivação intrínseca, $\alpha=0.83$ ).

Auto-conceito - Physical Self-Perception Profile $R$ (PSPP-R)

$E$ por fim, na terceira parte, o instrumento Physical Self-Perception Profile Revised (PSPP-R), traduzido para a língua portuguesa ${ }^{(27)}$ e que tem por base o questionário original de Fox $^{(11)}$. Constituído por setenta e quatro itens relativos ao autoconceito, para avaliar as 6 dimensões do autoconceito, competência desportiva, condição física, corpo atraente, força física, auto-estima corporal e auto estima global e avaliar a importância que os indivíduos atribuem às quatro dimensões, competência desportiva, condição física, corpo atraente e força física. Exemplo dos itens: "Eu faço bem todos os tipos de desportos". "Para si, o grau de importância que tem fazer bem todos os tipos de desportos é", cada um dos itens foi respondido numa escala Likert de quatro opções, nada verdadeiro, pouco verdadeiro, de certo modo verdadeiro ou realmente verdadeiro. A consistência 
interna das dimensões do auto-conceito foi boa (competência desportiva, $\alpha=0.85$; condição física, $\alpha=0.83$; corpo atraente, $\alpha=0.87$; força física, $\alpha=0.85$; auto-estima corporal, $\alpha=0.89$; auto-estima global, $\alpha=0.86$, importância da competência desportiva, $\alpha=0.75$; importância da condição física, $\alpha=0.76$; importância do corpo atraente, $\alpha=0.82$; importância da força física, $\alpha=0.82$; importância da auto-estima corporal, $\alpha=0.82$; importância da auto-estima global, $\alpha=0.81$ ).

\section{Procedimentos}

Para a selecção dos indivíduos foi contactado inicialmente o Conselho Executivo, para explicar o objectivo do estudo e autorizar a recolha de dados. O estudo foi aceite pela Comissão de Ética da Universidade Lusófona de Humanidades e Tecnologias, pelo que posteriormente foram contactados os professores do departamento de educação física, no sentido de definir a forma mais eficaz de aplicar os instrumentos a todas as turmas do ensino secundário da escola. Foi escolhida a semana de aplicação dos questionários e os professores disponibilizaram cerca de 30 minutos da aula de Educação Física. Os estudantes foram informados sobre o propósito do estudo, de que era de sua livre vontade participarem neste estudo. Após o seu consentimento para a participação, foi salientada a importância de respostas sinceras e de que estas seriam confidenciais e anónimas.

\section{Análise estatística}

Depois de aferida a normalidade dos dados, efectuou-se uma análise correlacional com a técnica produto-momento de Pearson, avaliando a o grau da associação entre a motivação e o auto-conceito. Para as comparações (e.g., género e grupos de prática - educação física, desporto escolar e desporto federado) foi efectuada uma MANOVA. Os valores das variáveis foram expressos através da média e desvio padrão. A significância estatística foi estabelecida para $p<0.05$. A análise estatística foi realizada com a aplicação informática SPSS 16.0.

\section{RESULTADOS}

A primeira análise avaliou o grau de associação entre as variáveis da motivação e auto-conceito, através do produto momento de Pearson, chegando-se aos resultados da Tabela 2 .
TABELA 2. Correlação de Pearson para análise da associação entre as dimensões da motivação e auto-conceito

\begin{tabular}{|c|c|c|c|c|c|c|}
\hline & \multicolumn{3}{|c|}{ Feminino } & \multicolumn{3}{|c|}{ Masculino } \\
\hline $\mathrm{N}(\%)$ & & $\begin{array}{c}237 \\
(51.5 \%)\end{array}$ & & & $\begin{array}{c}223 \\
(48.5 \%)\end{array}$ & \\
\hline \multirow[t]{2}{*}{ Idade $(M \pm D P)$} & & $16.49 \pm 1.04$ & & & $16.71 \pm 1.31$ & \\
\hline & $E F$ & EFMNF & EFD & $\mathrm{EF}$ & EFMNF & EFD \\
\hline $\mathrm{N}(\%)$ & $\begin{array}{c}150 \\
(32.6 \%)\end{array}$ & $50(10.9 \%)$ & $37(8.0 \%)$ & $91(19.8 \%)$ & $59(12.8 \%)$ & $73(15.9 \%)$ \\
\hline $\begin{array}{l}\text { Idade } \\
(M \pm D P)\end{array}$ & $16.45 \pm 1.05$ & $16.58 \pm 1.03$ & $16.51 \pm 1.07$ & $16.80 \pm 1.31$ & $16.61 \pm 1.46$ & $16.68 \pm 1.15$ \\
\hline
\end{tabular}

Verifica-se que as dimensões da motivação associadas aos factores mais extrínsecos (regulação externa), apresentaram valores de correlação negativos com o auto-conceito, no sentido em que os maiores valores de regulação externa estão associados aos menores valores de auto-conceito. Um cenário diametralmente oposto verifica-se com os tipos de motivação mais autodeterminados, com os maiores valores do autoconceito a estarem associados aos maiores valores das motivações identificada e intrínseca. Por último, verifica-se que a regulação introjectada se associa principalmente às dimensões da importância do auto-conceito.

Para analisar se existem diferenças no autoconceito em função dos grupos em estudo, foi efectuada uma MANOVA (Género x Grupo de Prática), chegando-se aos resultados desenhados na tabela 3.

TABELA 3. Média, desvio padrão e MANOVA para análise da interacção entre o género e o grupo de prática nas dimensões do auto-conceito

\begin{tabular}{|c|c|c|c|c|c|c|c|c|c|}
\hline \multirow[b]{2}{*}{ Variáveis } & \multicolumn{2}{|c|}{ a) $\mathrm{EF}$} & \multicolumn{2}{|c|}{ b) EFMNF } & \multicolumn{2}{|c|}{ c) EFD } & \multirow{2}{*}{$\begin{array}{c}\text { Género } \\
\mathrm{F}\end{array}$} & \multirow{2}{*}{$\begin{array}{c}\text { Grupo } \\
\mathrm{F}\end{array}$} & \multirow{2}{*}{$\begin{array}{c}\text { Género } x \\
\text { Grupo } \\
\mathrm{F}\end{array}$} \\
\hline & $M$ & $\mathrm{DP}$ & $M$ & $\mathrm{DP}$ & $M$ & DP & & & \\
\hline Competência Desportiva & & & & & & & & & \\
\hline Sexo feminino & 16.28 & 3.45 & 16.42 & 3.28 & 19.79 & 3.14 & $58.80^{\star \star \star}$ & $20.87^{\star \star *}$ & $4.15^{\star \star \star}$ \\
\hline Sexo masculino & 19.28 & 3.20 & 20.14 & 3.32 & 20.94 & 3.08 & & & \\
\hline Condição Física & & & & & & & & & \\
\hline Sexo feminino & 16.87 & 3.51 & 17.90 & 3.61 & 21.09 & 2.96 & $47.60^{\star \star *}$ & $36.32^{* * *}$ & 2.39 \\
\hline Sexo masculino & 19.60 & 3.22 & 21.11 & 3.02 & 22.35 & 3.32 & & & \\
\hline Corpo Atraente & & & & & & & & & \\
\hline Sexo feminino & 18.88 & 4.88 & 18.25 & 4.90 & 21.08 & 4.32 & $14.17^{\star * *}$ & 2.60 & $3.43^{*}$ \\
\hline Sexo masculino & 20.83 & 4.10 & 21.67 & 4.15 & 21.12 & 4.86 & & & \\
\hline Força Física & & & & & & & & & \\
\hline Sexo feminino & 14.64 & 3.47 & 14.94 & 3.51 & 17.74 & 3.44 & $63.95^{\star \star *}$ & $13.45^{\star \star *}$ & $4.11^{*}$ \\
\hline Sexo masculino & 17.78 & 3.40 & 19.23 & 3.86 & 19.18 & 3.67 & & & \\
\hline Auto-estima Corporal & & & & & & & & & \\
\hline Sexo feminino & 22.06 & 5.24 & 21.04 & 5.98 & 25.33 & 4.00 & $13.94^{\star \star \star}$ & $10.07^{\star \star *}$ & $3.37^{*}$ \\
\hline Sexo masculino & 23.90 & 4.00 & 24.61 & 3.89 & 25.45 & 4.43 & & & \\
\hline Auto-estima Global & & & & & & & & & \\
\hline Sexo feminino & 18.67 & 3.48 & 17.41 & 4.20 & 20.17 & 2.73 & 3.57 & $6.56^{\star \star}$ & $3.84^{*}$ \\
\hline Sexo masculino & 18.93 & 3.13 & 19.42 & 2.65 & 19.81 & 2.84 & & & \\
\hline
\end{tabular}

Os resultados revelam que os moços apresentam valores mais elevados de autoconceito corporal em todas as dimensões do PSPP-R (todos $p<0.001$ ), exceptuando a 
auto-estima global, onde não se registaram diferenças em função do género. Já nas comparações conforme o grupo de prática, verificou-se que os adolescentes que praticam desporto apresentam valores superiores de auto-conceito corporal quando comparados com os adolescentes dos restantes grupos (todos $\mathrm{p}<0.01$, teste post-hoc de Tukey), com excepção da dimensão corpo atraente, onde não se registaram diferenças. Quando se analisou a interacção entre as variáveis independentes, verificou-se uma interacção significativa entre o género e o grupo de prática nas variáveis competência desportiva $(F(2,443)=4.15, p<$ 0.05), corpo atraente $(F(2,443)=3.43, p<0.05)$, força física $(F(2,443)=4.11, p<0.05)$, auto-estima corporal $(F(2,443)=3.37, p<0.05)$ e auto-estima global $(F(2,443)=3.84, p<0.05)$. Apenas não se verificou interacção na dimensão condição física. Nas dimensões competência desportiva, corpo atraente, força física, auto-estima corporal e auto-estima geral esta interacção diz-nos que as diferenças são evidentes entre género em todos os grupos de prática, à excepção do grupo de desporto em que existe uma aproximação dos valores entre indivíduos do sexo masculino e feminino (ver FIGURA 1 para exemplo deste efeito interactivo).

Figura 1 - Interacção entre o género e o grupo de prática na dimensão auto-estima corporal

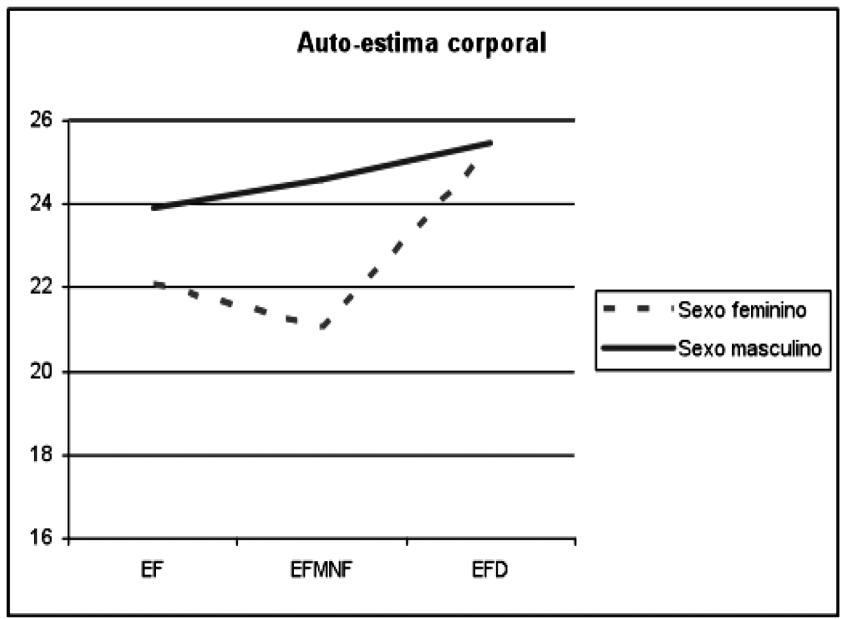

Seguidamente foi efectuada uma análise da interacção entre o género e o grupo de prática nas variáveis da importância do auto-conceito, chegandose aos resultados desenhados na tabela 4 .
TABELA 4. Média, desvio padrão e MANOVA para análise da interacção entre o género e o grupo de prática nas variáveis da importância do auto-conceito

\begin{tabular}{|c|c|c|c|c|c|c|c|c|c|}
\hline \multirow[b]{2}{*}{ Variáveis } & \multicolumn{2}{|c|}{ a) $\mathrm{EF}$} & \multicolumn{2}{|c|}{ b) MNF } & \multicolumn{2}{|c|}{ c) $D$} & \multirow{2}{*}{ Género } & \multirow{2}{*}{$\begin{array}{c}\text { Grupo } \\
\mathrm{F}\end{array}$} & \multirow{2}{*}{$\begin{array}{c}\begin{array}{c}\text { Género } x \\
\text { Grupo }\end{array} \\
F\end{array}$} \\
\hline & $\bar{M}$ & $\overline{D P}$ & $\bar{M}$ & $\overline{D P}$ & $\bar{M}$ & $\overline{\mathrm{DP}}$ & & & \\
\hline \multicolumn{10}{|l|}{ Importância da } \\
\hline \multicolumn{10}{|l|}{ Competência Desportiva } \\
\hline Sexo feminino & 15.40 & 2.90 & 15.44 & 2.89 & 17.46 & 2.68 & $8.13^{* *}$ & $13.78^{\star \star \star}$ & 1.59 \\
\hline Sexo masculino & 16.01 & 3.22 & 17.14 & 3.02 & 17.81 & 3.02 & & & \\
\hline \multicolumn{10}{|l|}{ Importância da } \\
\hline \multicolumn{10}{|l|}{ Condição Física } \\
\hline Sexo feminino & 17.38 & 2.77 & 17.94 & 2.65 & 19.57 & 2.41 & 0.22 & $22.74^{\star * *}$ & 0.26 \\
\hline Sexo masculino & 17.27 & 3.09 & 18.29 & 2.80 & 19.74 & 2.86 & & & \\
\hline \multirow{2}{*}{\multicolumn{10}{|c|}{$\begin{array}{l}\text { Importância do } \\
\text { Corpo Atraente }\end{array}$}} \\
\hline & & & & & & & & & \\
\hline Sexo feminino & 18.94 & 4.10 & 19.68 & 4.21 & 19.31 & 3.17 & 1.26 & 2.65 & 0.56 \\
\hline Sexo masculino & 17.99 & 4.22 & 19.12 & 3.89 & 19.40 & 4.10 & & & \\
\hline \multicolumn{10}{|l|}{$\begin{array}{l}\text { Importância da } \\
\text { Força Física }\end{array}$} \\
\hline Sexo feminino & 13.69 & 2.91 & 13.59 & 2.90 & 15.16 & 2.09 & $37.28^{\star \star \star}$ & $5.59^{\star *}$ & 0.90 \\
\hline Sexo masculino & 15.55 & 3.85 & 16.26 & 3.33 & 16.69 & 3.25 & & & \\
\hline
\end{tabular}

Nota: ${ }^{* *} p<.01 ;{ }^{* * *} p<.001$. EF - Educação Física; EFMNF - Educação Física mais Modalidade Desportiva Não Federada; EFD - Educação Física mais Desporto

A análise das diferenças da importância dada às dimensões do auto-conceito corporal em função do género, verificou-se que os moços consideram mais importante a competência desportiva e força física $(p<0.01)$ comparativamente às moças. Os praticantes de desporto deram mais importância à competência desportiva, condição física e força física do que os adolescentes dos restantes grupos de prática ( $p<0.001$, teste post-hoc Tukey). Nas dimensões relacionadas com a importância do auto-conceito não se verificaram interacções significativas.

Seguidamente, foi efectuada uma análise semelhante às anteriores, mas tendo a motivação como variável dependente (TABELA 5).

TABELA 5. Média, desvio padrão e MANOVA para análise da interacção entre o género e o grupo de prática nas variáveis da motivação

\begin{tabular}{|c|c|c|c|c|c|c|c|c|c|}
\hline Variáveis & $\bar{M}$ & $\mathrm{DP}$ & $\bar{M}$ & $\mathrm{DP}$ & $\bar{M}$ & $\mathrm{DP}$ & $\bar{F}$ & $\bar{F}$ & $F$ \\
\hline \multicolumn{10}{|l|}{ Amotivação } \\
\hline $\begin{array}{l}\text { Sexo feminino } \\
\text { Sexo masculino }\end{array}$ & 0.88 & 2.09 & 0.69 & 1.62 & 0.40 & 1.19 & 0.60 & $4.94^{* *}$ & 0.94 \\
\hline \multicolumn{10}{|l|}{ Regulação Externa } \\
\hline Sexo feminino & 1.74 & 2.40 & 2.12 & 2.19 & 0.94 & 1.93 & 0.01 & $5.09^{* *}$ & 0.64 \\
\hline Sexo masculino & 1.95 & 2.88 & 1.72 & 2.28 & 1.08 & 1.68 & & & \\
\hline \multicolumn{10}{|l|}{ Regulação Introjectada } \\
\hline Sexo feminino & 3.31 & 2.72 & 3.63 & 2.84 & 4.56 & 3.20 & 2.25 & $4.97^{\star \star}$ & 0.09 \\
\hline Sexo masculino & 2.94 & 2.85 & 3.29 & 2.72 & 3.91 & 3.14 & & & \\
\hline \multicolumn{10}{|l|}{ Regulação Identificada } \\
\hline Sexo feminino & 10.24 & 2.83 & 11.49 & 3.00 & 13.14 & 2.21 & 13.97 & $37.30^{\star \star *}$ & 0.06 \\
\hline Sexo masculino & 10.60 & 2.78 & 12.02 & 3.04 & 13.42 & 2.19 & & & \\
\hline \multicolumn{10}{|l|}{ Motivação Intrínseca } \\
\hline Sexo feminino & 11.10 & 3.70 & 12.12 & 3.23 & 15.17 & 1.50 & 3.46 & $40.91^{* * *}$ & $4.48^{*}$ \\
\hline Sexo masculino & 11.81 & 3.29 & 13.93 & 2.47 & 14.42 & 1.95 & & & \\
\hline
\end{tabular}

Nota: ${ }^{*} \mathrm{p}<.05 ;{ }^{* *}<.01 ;{ }^{* * *} \mathrm{p}<.001, \mathrm{EF}$ - Educação Física; EFMNF - Educação Física mais Modalidade Desportiva Não Federada; EFD - Educação Física mais Desporto

Não se registaram diferenças nos tipos de motivação conforme o género. Pelo contrário, o grupo de adolescentes que pratica desporto apresentou valores inferiores de amotivação e regulação externa, e superiores de motivação 
introjectada quando comparado com os restantes grupos $(p<0.05$, teste post-hoc Tukey). Na motivação intrínseca e regulação identificada verificou-se que o grupo de adolescentes que pratica desporto apresentou os valores mais elevados de todos os grupos, e que o grupo que pratica modalidades não federadas apresentou valores mais elevados do que o grupo que só faz Educação Física ( $p<0.001$, teste post-hoc Tukey). Não se verificaram interacções significativas entre o género e o grupo de prática nas variáveis relacionadas com a motivação, à excepção da variável motivação intrínseca $(F(2,443)=40.91$, $p<0.05)$. Esta interacção na dimensão motivação intrínseca diz-nos que existe uma aproximação de valores entre género no grupo educação física (FIGURA 2). No grupo dos adolescentes que praticam modalidade não federada a motivação intrínseca é superior nos indivíduos do sexo masculino comparativamente aos do sexo feminino, enquanto que no grupo de desporto esta situação inverte-se, já que agora são os indivíduos do sexo feminino que apresentam maior motivação intrínseca.

Figura 2 - Interacção entre o género e o grupo de prática na variável da motivação intrínseca

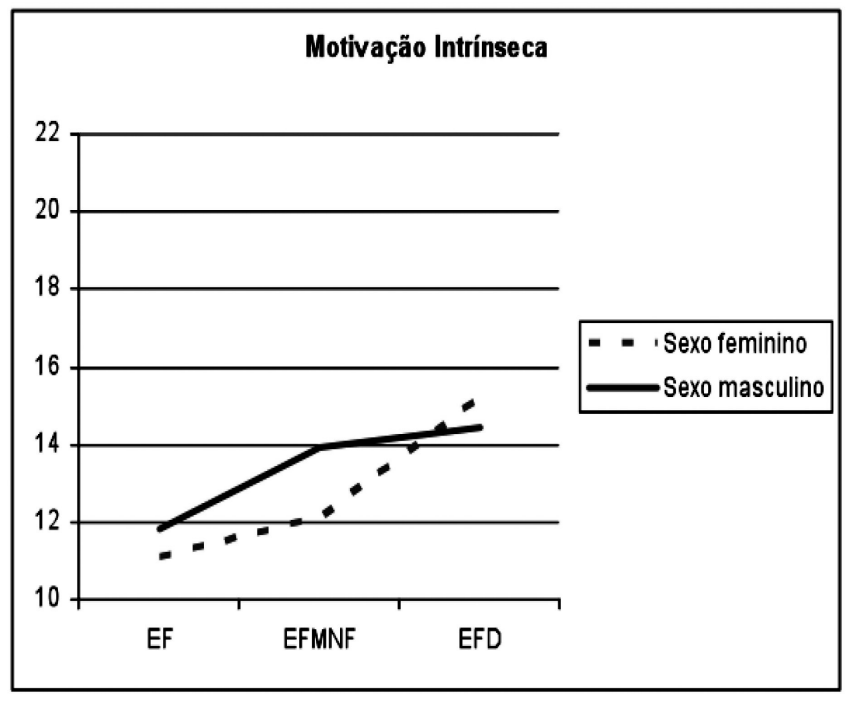

\section{DISCUSSÃO}

Com este estudo pretendemos analisar o autoconceito e a importância atribuída ao auto-conceito em adolescentes, estudando a sua associação com a motivação para a prática de actividade física e verificando se varia consoante o nível de actividade física e género.

Através dos resultados do nosso estudo verificamos que quanto maior o nível de prática, mais elevado é o auto-conceito, a importância atribuída às dimensões do auto-conceito e à motivação. O grupo de indivíduos que praticam desporto tem valores significativamente superiores aos restantes grupos na competência desportiva e importância atribuída, força física e importância atribuída, auto estima corporal e auto-estima global, talvez devido ao grupo de desporto ser constituído por um conjunto de indivíduos que por estarem num nível competitivo já foram seleccionados por serem melhores a nível desportivo, tendo assim valores superiores de competência, força e importância atribuída ou pelo facto de treinarem com mais frequência semanal devido ao nível onde se encontram, proporcione melhorias nestas dimensões, visto que o aumento da actividade física tem um impacto positivo na auto percepção do domínio atlético ${ }^{(14)}$. Parece-nos uma consequência lógica que os valores elevados obtidos nas dimensões de competência desportiva e força física contribuam para os valores elevados de auto-estima corporal que está num patamar superior, pois refere-se ao modo como os indivíduos se sentem em relação à sua dimensão física em geral e por sua vez influencia positivamente a autoestima global. Estes resultados são semelhantes ao estudo de Schneider et al, onde se verificou que a realização de actividade física melhorou a autoestima corporal dos participantes ${ }^{(14)}$.

No entanto, nos outros dois grupos, o grupo de indivíduos que só fazia educação física e o que praticava modalidade não federada, não se encontraram diferenças significativas nas dimensões referidas anteriormente, competência desportiva e importância atribuída, força física e importância atribuída, auto-estima corporal e auto-estima global, o que talvez possa ter ocorrido devido à modalidade não federada ser vista pelos indivíduos como mais uma actividade além da Educação Física, que apenas realizam com 1 ou 2 vezes semanalmente e que por isso não provoque diferenças na sua competência desportiva ou força física, bem como auto-estima corporal e autoestima global. No entanto, esta suposição já não é válida para a dimensão da condição física, pois os resultados indicam diferenças em todos os grupos de prática, apresentando o grupo de desporto 
valores mais altos e o grupo de educação física os valores mais baixos, o que de acordo com os princípios do treino nos parece evidente, quem treina com mais regularidade tem melhor condição física e talvez por esse motivo os indivíduos tenham uma auto-percepção correcta da sua condição física.

Os nossos resultados mostraram que os adolescentes que praticam desporto estão mais motivados por regulação identificada e motivação intrínseca do que os que praticam modalidades não federadas, que por sua vez estão mais motivados por regulação identificada e motivação intrínseca que os que apenas praticam educação física. Segundo a literatura, os motivos intrínsecos estão associados a consequências psicológicas positivas e grande adesão ao exercício ${ }^{(28)}$, o que nos leva a sugerir que a maioria dos indivíduos pertencentes ao grupo de desporto no nosso estudo, para se encontrarem motivados positivamente, realizam o desporto com prazer e tentam a superação deles próprios ${ }^{(21)}$ e também por serem ainda adolescentes, talvez tenham também uma boa estrutura social e ambiental à volta da actividade física (ex. acesso facilitado ao desporto, encorajamento positivo por parte dos pais e professores) e maior apoio das pessoas envolventes, que possam contribuir para uma maior motivação(21).

Por sua vez, o grupo de indivíduos que apenas pratica educação física e o grupo de indivíduos que pratica uma outra modalidade não federada apresentaram valores elevados de amotivação e regulação externa. Esta ausência de motivação poderá significar que estes indivíduos se sintam incompetentes para a realização da actividade ou porque não têm vontade da realização da mesma, ou porque como a Educação Física é uma disciplina obrigatória em Portugal possa comprometer a motivação intrínseca, dado que os alunos não possuem possibilidades de escolha(22). Em relação ao grupo de indivíduos que também praticam uma outra modalidade não federada, os valores elevados de amotivação e motivação por regulação externa poderão predizer que realizam a actividade, não como uma forma de divertimento ou prazer, mas como um modo de obter recompensas ou evitar consequências negativas ${ }^{(21,22,24,28)}$.

Estes resultados levam-nos a sugerir que existe uma relação entre estes constructos, como indicam os autores Moreno e Cervelló(13), quanto maior o nível de actividade física melhores as auto-percepções corporais. Também num estudo efectuado com o propósito de examinar as relações entre os motivos para o exercício e a auto-estima corporal, os autores verificaram que os indivíduos que apresentaram valores mais elevados de motivação por regulação identificada e intrínseca indicaram ter percepções mais elevadas da sua auto-estima corporal(13), o que apoia os resultados do nosso estudo, pois verificámos que os maiores valores do auto-conceito se encontram associados aos maiores valores das motivações identificada e intrínseca.

Verificámos também que os indivíduos do sexo masculino apresentam valores superiores de auto-conceito corporal, de importância atribuída às dimensões do auto-conceito e motivação em relação aos indivíduos do sexo feminino, o que vai de encontro aos resultados de outros autores ${ }^{(12)}$. Provavelmente, esta situação verifica-se devido às dimensões do auto-conceito focarem competências específicas (como por exemplo competência desportiva, condição física, força física) onde os indivíduos do sexo masculino são vistos como tendo mais competências físicas que as moças $^{(7)}$. Por outro lado, a ausência de diferenças na importância dada ao corpo atraente podem derivar das alterações corporais características da adolescência, levando a que ambos os géneros, embora por razões opostas (ganho de massa muscular nos moços - com um sentido positivo face a um ideal corporal masculino; e ganho de massa gorda nas moças - com um sentido negativo face ao ideal feminino), indiquem valores semelhantes nas suas respostas ${ }^{(5,6)}$.

Verificámos que a percepção corporal estava associada aos tipos de motivação para a participação que os indivíduos adoptam. Wilson e Rodgers(19), verificaram que os indivíduos que apresentaram valores mais elevados de motivação por regulação identificada e intrínseca indicaram ter percepções mais elevadas da sua auto-estima corporal. Em vários estudos foi reportado que entre os indivíduos do sexo feminino, a prática de exercício como objectivo de aparência corporal/ manutenção do peso corporal está associada a um grande descontentamento corporal, menor satisfação corporal, a um maior distúrbio da imagem corporal e a efeitos corporais negativos ${ }^{(15,16)}$.

Consideramos importante assinalar

que 
este estudo teve como limitações a utilização do questionário PSPP-R que, embora apresente excelentes características psicométricas nos trabalhos prévios de validação, ainda não se encontra publicado (foi submetido a uma revista internacional e foi aceite propondo-se pequenas revisões). Ainda relativo aos questionários salientese que o BREQ-2 apresentou características psicométricas abaixo das que resultaram do estudo de validação, pese embora possam ser consideradas aceitáveis com reservas, visto que cada dimensão inclui um número pequeno de items (o valor de >.60 tem sido sugerido para estas situações como aceitável ${ }^{(29)}$. A criação dos grupos de estudo poderá ser igualmente considerada uma limitação, pois considerámos a prática de desporto sem olharmos para os diferentes níveis competitivos, obviamente associados a cargas de treino e envolvimento diferenciados. O mesmo poderá ter acontecido com o grupo de modalidades não federadas, pois não se conseguiu diferenciar o envolvimento dos sujeitos na prática, tendo-se verificado uma grande dispersão de modalidades referidas pelos adolescentes, que obviamente representam um contexto e vivências diferenciadas que podem influenciar de forma díspar o autoconceito corporal e a qualidade da motivação.

Concluindo, verificámos através dos resultados do nosso estudo, que quanto maior o nível de prática, mais elevados são os valores de autoconceito, importância atribuída às dimensões do auto-conceito e motivação, bem como os indivíduos do sexo masculino apresentam valores superiores nestes parâmetros estudados face aos indivíduos do sexo feminino. O que nos leva a realçar a importância de proporcionarmos uma boa estrutura social e ambiental à volta da actividade física desde tenra idade, permitindo o acesso facilitado à actividade física e ao desporto, um encorajamento positivo por parte da família, educadores, professores e treinadores e tentar que a actividade seja realizada com prazer, aumentando a motivação e contribuindo para uma melhor auto-estima.

\section{REFERÊNCIAS BIBLIOGRÁFICAS}

1. AAP, CSMFCSH. Active Healthy Living: Prevention of Childhood Obesity Through Increased Physical Activity Pediatrics. 2006;117(5):1834-42.
2. Wang C, Biddle S. Understanding Young People's Motivation Toward Exercise. An Integration of Sport Ability Beliefs, Achievement Goal Theory, and Self Determination Theory,. In: Hagger M, Chatzisarantis N, editors. Intrinsic Motivation and Self-Determination in exercise and Sport. Champaign, IL: Human Kinetics; 2007. p. 193-208.

3. IDP. Livro Verde da Actividade Física e Obesidade em Portugal in prep.

4. WHO. Inequalities in Young People's Health HBSC International Report from the 2005/2006 Report. Currie C, editor2008.

5. Striegel-Moore RH, Silberstein LR, Rodin J. Toward an understanding of risk factors for bulimia. Am Psychol. 1986 Mar;41(3):246-63.

6. Rolls B, Federoff I, Guthrie J. Gender differences in eating behaviour and body weigth regulation. Health Psy. 1991;10:133-42.

7. Fox KR. The physical self: From motivation to well-being. Champaign, Illinois: Human Kinetics; 1997.

8. Buckworth J, Dishman R. Exercise Psychology. Champaign, IL: Human Kinetics; 2002.

9. Marsh HW, Papaioannou A, Theodorakis Y. Causal ordering of physical self-concept and exercise behavior: reciprocal effects model and the influence of physical education teachers. Health Psychol. 2006 May;25(3):316-28.

10. Marsh HW, Shavelson R. Self-concept: Its multifaceted, hierarquical structure. Educational Psychologist. 1985;20:107-25.

11. Fox KR. The Physical Self-Perception Profile manual. DeKalb, IL: Northern Illinois University; 1990.

12. Hagger $M$, Biddle $S$, Wang C. Physical Self-Concept in Adolescence: Generalizability of a Multidimensional, Hierarchical Model Across Gender and Grade. Educational and Psychological Measurement. 2005;65(2):297-322.

13. Moreno J, Cervelló E. Physical Self-Perception in Spanish Adolescents: Effects of Gender and involvement in physical activity. Journal of Human Movement Studies. 2005;48:291-311.

14. Schneider M, Dunton GF, Cooper DM. Physical Activity and Physical Self-Concept among Sedentary Adolescent Females; An Intervention Study. Psychol Sport Exerc. 2008 Jan;9(1):1-14.

15. Cash TF, Novy PL, Grant JR. Why do women exercise? Factor analysis and further validation of the Reasons for Exercise Inventory. Percept Mot Skills. 1994 Apr;78(2):539-44. 
16. McDonald K, Thompson J. Eating disturbance, body image dissatisfaction and reasons for exercising: Gender differences and correlational findings. Int J Eat Disorders. 1992;11:289-92.

17. Ingledew D, Sullivan G. Effects of body mass and body image on exercise motives in adolescence. Psychol Sport Exerc. 2002;3:323-38.

18. Hein V, Hagger MS. Global self-esteem, goal achievement orientations, and self-determined behavioural regulations in a physical education setting. J Sports Sci. 2007 Jan 15;25(2):149-59.

19. Wilson P, Rdgers W. The Relationship Between Exercise Motives and Physical Self-Esteem in Female Exercise Participants: An Application of SelfDetermination Theory. Journal of Applied Biobehavioral Research. 2002;7(1):30-43.

20. Ryan R, Deci E. Active Human Nature: Self-Determination Theory and the promotion and Maintenance of Sport, Exercise and Health. In: Hagger $\mathrm{M}$, Chatzisarantis $\mathrm{N}$, editors. Intrinsic Motivation and Self-Determination in exercise and Sport. Champaign, IL: Human Kinetics; 2007. p. 1-19.

21. Vansteenkiste M, Soenens B, Lens W. Intrinsic Versus Extrinsic Goal Promotion in Exercise and Sport: Understanding the Differential Impacts on Performance and Persistence. In: Hagger M, Chatzisarantis N, editors. Intrinsic Motivation and Self-Determination in exercise and Sport. Champaign, IL: Human Kinetics; 2007. p. 167-208.

22. Ntoumanis N. A self-determination approach to the understanding of motivation in physical education. Br J Educ Psychol. 2001 Jun;71(Pt 2):225-42.

23. Deci EL, Ryan RM. Intrinsic motivation and selfdetermination in human behavior. New York: Plenum; 1985.
24. Thogersen-Ntoumani $\mathrm{C}$, Ntoumanis $\mathrm{N}$. The role of self-determined motivation in the understanding of exercise-related behaviours, cognitions and physical self-evaluations. J Sports Sci. 2006 Apr;24(4):393-404.

25. Markland DA, Tobin V. A modification to the Behavioural Regulation in Exercise Questionnaire to include and assessment of Amotivation. Journal of Sport and Exercise Psychology. 2004;26:191-6.

26. Palmeira AL, Teixeira PJ, Silva MN, Markland D, editors. Confirmatory Factor Analysis of the Behavioural Regulation in Exercise Questionnaire - Portuguese Version. FEPSAC 2007; 2007; Halkidiki, Greece.

27. Lindwall $M$, Asçi HF, Hagger $M$, Palmeira $A L$, Fox KR. The Importance of Importance in the Physical Self: Support for the Theoretically Appealing but Empirically Elusive Model of James. Journal Social and Personality Psychology. submitted.

28. Markland D, Ingledew D. Exercise Participation Motives: a Self-Determination Theory Perspective. In: Hagger M, Chatzisarantis N, editors. Intrinsic Motivation and Self-Determination in Exercise and Sport. Champaign, IL: Human Kinetics; 2007. p. 23-34.

29. Morgan GA, Griego OV. Easy Use and Interpretation of SPSS for Windows. New Jersey: Lawrence Erlbaum Associates; 1998.

Universidade Lusófona de Humanidades e Tecnologias Faculdade de Educação Física e Desporto

Campo Grande, 376

1749-024, Lisboa - Portugal

TIf: +3512175155580

Mónica Silva - e-mail: monica.sincro@gmail.com António Labisa Palmeira (autor para correspondência) e-mail: antonio.palmeira@ulusofona.pt 